OPEN ACCESS

Edited by:

Changlian Zhu,

Third Affiliated Hospital of Zhengzhou

University, China

Reviewed by:

Rosa Marotta

University of Magna Graecia, Italy

Jakub Mieczkowski,

Medical University of Gdansk, Poland

*Correspondence:

Fuquan Zhang

zhangfq@nimu.edu.cn

Hongbao Cao

caohon2010@gmail.com

Specialty section:

This article was submitted to

Pediatric Neurology,

a section of the journal

Frontiers in Neurology

Received: 09 January 2020

Accepted: 22 May 2020

Published: 15 July 2020

Citation:

Xie Q, Li Z, Wang Y, Zaidi S, Baranova A, Zhang $\mathrm{F}$ and $\mathrm{Cao} H$ (2020) Preeclampsia Drives Molecular Networks to Shift Toward Greater Vulnerability to the Development of

Autism Spectrum Disorder.

Front. Neurol. 11:590.

doi: 10.3389/fneur.2020.00590

\section{Preeclampsia Drives Molecular Networks to Shift Toward Greater Vulnerability to the Development of Autism Spectrum Disorder}

\author{
Qinglian Xie ${ }^{1}$, Zhe $\mathrm{Li}^{2}$, Yan Wang ${ }^{1}$, Shan Zaidi ${ }^{3}$, Ancha Baranova ${ }^{3,4}$, Fuquan Zhang ${ }^{5 *}$ and \\ Hongbao $\mathrm{CaO}^{3,6 *}$ \\ ${ }^{1}$ Department of Outpatient, West China Hospital of Sichuan University, Chengdu, China, ${ }^{2}$ Mental Health Center and National \\ Clinical Research Center for Geriatrics, West China Hospital of Sichuan University, Chengdu, China, ${ }^{3}$ School of Systems \\ Biology, George Mason University, Fairfax, VA, United States, ${ }^{4}$ Research Centre for Medical Genetics, Moscow, Russia, \\ ${ }^{5}$ Department of Psychiatry, The Affiliated Brain Hospital of Nanjing Medical University, Nanjing, China, ${ }^{6}$ Department of \\ Psychiatry, First Hospital/First Clinical Medical College of Shanxi Medical University, Taiyuan, China
}

Preeclampsia (PE) confers a significant risk for subsequent diagnosis with autism spectrum disorder (ASD), with the mechanisms underlying this observation being largely unknown. To identify molecular networks affected by both PE and ASD, we conducted a large-scale literature data mining and a gene set enrichment analysis (GSEA), followed by an expression mega-analysis in 13 independently profiled ASD datasets. Sets of genes implicated in ASD and in PE significantly overlap (156 common genes; $p=3.14 \mathrm{E}^{-67}$ ), with many biological pathways shared (94 pathways; $\left.p<1.00 \mathrm{E}^{-21}\right)$. A set of PE-driven molecular triggers possibly contributing to worsening the risk of subsequent ASD was identified, possibly representing a regulatory shift toward greater vulnerability to the development of ASD. Mega-analysis of expression highlighted RPS4Y1, an inhibitor of STAT3 that is expressed in a sexually dimorphic manner, as a contributor to both PE and ASD, which should be evaluated as a possible contributor to male predominance in ASD. A set of PE-driven molecular triggers may shift the developing brain toward a greater risk of ASD. One of these triggers, chromosome $Y$ encoded gene RPS4Y1, an inhibitor of STAT3 signaling, warrants evaluation as a possible contributor to male predominance in ASD.

Keywords: autism, autism spectrum disorder, preeclampsia, GSEA, extreme male brain theory

\section{INTRODUCTION}

Autism spectrum disorders (ASD) are a range of neurodevelopmental mental disorders affecting nearly $1 \%$ of the global population ( 62.2 million globally) (1) and more than $2 \%$ of children (about 1.5 million) in the United States of America (www.hrsa.gov). Studies in recent years revealed hundreds of genes linked to ASD, paving the way for understanding the pathological mechanisms of the disease (2-4).

Preeclampsia (PE) is a disorder of pregnancy that increases the risk of poor outcomes for both the newborn and the mother. In PE, patients commonly present with high blood pressure, then their condition aggravates with the reduction in utero placental blood flow (1). Recent studies indicate that PE is one of the important risk factors for $\operatorname{ASD}(5,6)$. However, the underlying mechanisms are largely unknown. 
ASD and PE do share at least some pathophysiological pathways. For example, one of the core clinical features of PE is a systemic inflammation in a mother (Shennan et al., 2015). In turn, prenatal exposure to inflammation leads to exaggerated stress response and cytokine expression in a newborn (7). As these changes persist long-term, they may undermine normal postnatal development of the brain (8), increasing the chances for subsequent ASD diagnosis. It is likely, however, that the molecular networks connecting $\mathrm{PE}$ and ASD are not limited to relatively non-specific inflammatory signaling. Identification of the shared pathways may aid in understanding the mechanisms of PE-dependent delay in brain development or its contribution to ASD-specific defects while providing possible therapeutic targets involved in the pathogenesis of both diseases.

To dissect the association between PE and ASD at the genetic level, we employed the Pathway Studio (www.pathwaystudio. com) knowledge database to undertake large-scale literature mining effort and integrated its results with an analysis of multiple PE and ASD expression datasets. We identified a set of PE-driven molecular triggers, possibly contributing to worsening the risk of subsequent ASD through a regulatory shift toward greater vulnerability to the development of ASD. Chromosome Y-encoded gene RPS4Y1, an inhibitor of STAT3 signaling, was highlighted as a PE-driven contributor to ASD phenotypes. RPS4Y1 warrants evaluation as a possible contributor to male predominance in ASD.

\section{MATERIALS AND METHODS}

This study utilized the following workflow. First, the molecules involved in either PE or ASD were extracted in the Pathway Studio environment; common molecules and pathways connecting with both diseases were identified. All data and analysis results were organized in a database ASD_PE. The downloadable form of these two databases is available at gousinfo.com/database/Data_Genetic/ASD_PE.xlsx. Then, each PE-specific gene was tested in expression mega-analysis performed across 13 independently obtained, publicly available ASD datasets retrieved from Gene Expression Omnibus (GEO) (https://www.ncbi.nlm.nih.gov/geo/). Thereafter, functional network analysis was performed to study the pathogenic significance of identified genes for ASD-related processes.

\section{Disease-Gene Relation Data}

Disease-gene relation data for both ASD and PE were acquired through large-scale literature data analysis assisted by the Pathway Studio environment (www.pathwaystudio.com) commonly utilized for modeling the relationships between proteins, genes, complexes, cells, tissues, and diseases (9). Extracted relation data were uploaded in ASD_PE. For each of the genes linked to any of these two diseases, supporting references were examined and collated (ASD_PE: Ref4PE; Ref4ASD), including titles of the references and the related sentences describing the disease-gene relationship. Fisher's exact test was employed to compare the significance of the overlap between ASD genes and PE genes (https://david.ncifcrf.gov/ content.jsp?file=functional_annotation.html).
TABLE 1 | A summary of the datasets utilized in expression mega-analysis.

\begin{tabular}{llccl}
\hline Study Name & Dataset GEOID & \#Control & \#Case & Country \\
\hline Nishimura et al. (2007) & GSE7329 & 15 & 15 & USA \\
Hu et al. (2009) & GSE15402 & 29 & 26 & USA \\
Hu et al. (2009) & GSE15402 & 29 & 30 & USA \\
Hu et al. (2009) & GSE15451 & 17 & 21 & USA \\
Alter et al. (2011) & GSE25507 & 64 & 82 & USA \\
Kuwano et al. (2011) & GSE26415 & 42 & 21 & Japan \\
Voineagu et al. (2011) & GSE28521 & 40 & 39 & USA \\
Colak and Kaya, (2014) & GSE29691 & 13 & 2 & Saudi Arabia \\
Luo et al. (2012) & GSE37772 & 206 & 233 & USA \\
Ginsberg et al. (2012) & GSE38322 & 18 & 18 & USA \\
Pramparo et al. (2015) & GSE42133 & 56 & 91 & USA \\
Griesi-Oliveira et al. & GSE62632 & 12 & 6 & USA \\
(2014) & & & & \\
François et al. (2014) & GSE63524 & 5 & 6 & France \\
Liu et al. (2017) & GSE65106 & 38 & 21 & USA \\
\hline
\end{tabular}

\section{Mega-Analysis of Expression Datasets}

To compile the list of gene expression datasets, a publicly available GEO database was searched using keywords "autism spectrum disorder" and "ASD," which has returned 321 entries. This search covered the entire content of GEO and had no selection bias. Further filtering was performed according to the following criteria: (1) The data type is RNA expression; (2) the study design is case vs. control; (3) the original datasets and format files are available; and (4) the sample organism is Homo sapiens. A total of 14 datasets satisfied all the criteria listed and were pipelined into the mega-analysis of expression patterns as raw data files (Table $\mathbf{1}$ ).

For across-dataset mega-analysis, the expression data were normalized and log2-transformed. The mega-analysis workflow pools individual gene expression measurements while correcting for between-study variation (10). For each expression dataset, the log-fold change (LFC) in ASD samples was calculated and used as the index of effect size. Both the fixed-effect model and randomeffects model (11) were tested to study the effect size of PE-related genes on ASD, and their outputs compared. For each model, the heterogeneity of study inputs was calculated. Analyses were conducted using MATLAB (R2017a) mega-analysis package. To note, we used the term "mega-analysis" instead of "meta-analysis" to address the fact that the LFC of expression for each gene was calculated from the original datasets rather than using the values extracted from existing publications, which is the major difference between the two terms.

In mega-analysis, each gene was evaluated according to the following criteria: (1) The results were calculated from at least half of the studies; (2) $p<0.05$; and (3) effect size (LFC) $>0.59$ or $<-1.00$. When a gene has an effect size LFC $>0.59$ or $<$ -1.00 , it means that the change in the expression level of the gene had increased by more than $50 \%$ or decreased by more than $50 \%$. While ASD_PE $\rightarrow$ Mega-analysis presents mega-analysis outputs for the entire set of the analyzed genes, here we will discuss only the genes that satisfy the significance criteria outlined above. 
TABLE 2 | Genetic pathways enriched with 156 genes contributing to both preeclampsia and ASD.

\begin{tabular}{lccccc}
\hline Name & GO ID & $\begin{array}{c}\text { \# of } \\
\text { Entities }\end{array}$ & Overlap & p-value \\
\hline GO: response to toxic substance & 0009636 & 634 & 52 & $4.86 \mathrm{E}-38$ \\
GO: response to extracellular stimulus & 0009991 & 761 & 53 & $1.61 \mathrm{E}-35$ \\
GO: response to nutrient levels & 0031667 & 730 & 52 & $2.16 \mathrm{E}-35$ \\
GO: positive regulation of locomotion & 0040017 & 666 & 49 & $8.86 \mathrm{E}-34$ \\
GO: positive regulation of cell motility & 2000147 & 630 & 48 & $8.87 \mathrm{E}-34$ \\
GO: aging & 0016280 & 493 & 44 & $1.43 \mathrm{E}-33$ \\
GO: positive regulation of cell migration & 0030335 & 603 & 47 & $1.47 \mathrm{E}-33$ \\
GO: positive regulation of cellular & 0051272 & 650 & 48 & $2.4 \mathrm{E}-33$ \\
component movement & & & & \\
GO: regulation of response to external & 0032101 & 977 & 55 & $6.88 \mathrm{E}-33$ \\
stimulus & & & & \\
GO: regulation of secretion & 0051046 & 985 & 54 & $1.25 \mathrm{E}-31$ \\
\hline
\end{tabular}

For each pathway/GO term, the p-value was calculated using the Fisher exact test against the hypothesis that a randomly selected gene group of the same size $(N=156)$ can generate a similar or higher overlap with the corresponding pathway/GO term. All these pathways/GO terms passed the FDR correction ( $q=0.05$ )

\section{Gene Set Enrichment Analysis (GSEA) and Shortest Path Analysis}

To gain functional insights into genes implicated both in ASD and in PE, we conducted a gene set enrichment analysis (GSEA) in the Pathway Studio environment, with enrichment $p$-values being corrected according to the Benjamini-Hochberg procedure (12). In addition to GSEA, for a gene showing significance in the mega-analysis, which was not yet described as an ASD contributor, a literature-based functional pathway analysis was conducted using the "Shortest Path" module of Pathway Studio (www.pathwaystudio.com).

\section{Multiple Linear Regression Analysis}

A multiple linear regression analysis was employed to study the possible influence of the following three factors on the gene expression change: sample size, population region, and study date. $P$-values and $95 \%$ confidence interval (CI) were reported for each of these factors. The analysis was performed in Matlab (R 2017a) with the "regress" statistical analysis package.

\section{RESULTS}

\section{Common Genes for PE and ASD}

As could be seen in the ASD_PE database, a total of 1,188 genes were associated with PE (see ASD_PE: PE_Genes and Ref4PE), and a total of 624 genes were associated with ASD (ASD_PE: ASD_Genes and Ref4ASD), with a significant overlap $(N=156$, Fisher's exact test $p=3.14 \mathrm{E}-67$; ASD_PE: common genes). To investigate the pathways shared by $\mathrm{PE}$ and ASD, a set of 156 common genes associated with both ASD and PE was submitted to a Gene Set Enrichment Analysis (GSEA) executed by using Pathway Studio. A total of 116 out of these 156 genes were shared among the top 10 most significantly enriched pathways $(p<$ 1.20E-31, $q=0.05$ for FDR), which are presented in Table 2 .
The full 94 pathways/gene sets enriched with $p<1.00 \mathrm{e}-20$, which encompassed 144 out of 156 genes, were presented in ASD_PE $\rightarrow$ Common Pathways. Notably, a majority of the shared pathways highlighted by the GSEA approach were related to responses to some kind of external stimuli, including toxic ones, and to various aspects of the migration of cells. For detailed information regarding these significantly enriched pathways, please refer to ASD_PE $\rightarrow$ Common Pathways.

We also identified six positive regulators for ASD that have been stimulated by PE condition and one inhibitor (IGF1) that has been deactivated during PE (Figure 1). The detailed information of the pathway presented in Figure 1 can be found in ASD_PE $\rightarrow$ Prognostic pathway, including the type of the relationship, supporting references, and related sentences from the references where the relationship has been identified.

\section{Co-directionality of the PE and ASD Phenotype Interaction}

Pathway analysis has also identified multiple molecules influenced by both PE and ASD (Figure 2). Notably, these interactions were predominantly co-directional. A total of 25 molecules upregulated in ASD were stimulated in PE, and a total of 13 molecules suppressed in ASD were negatively affected by PE. The detailed information regarding the network presented in Figure 2 can be found in ASD_PE $\rightarrow$ Diagnostic pathway, with each network-related entry including the type of the relationship, supporting references, and related sentences from the references where the relationship has been identified.

\section{Mega-Analysis of Gene Expression Pattern Identified RPS4Y1 as a Novel Contributor to ASD}

Only one gene, RPS4Y1, has satisfied the significance criteria outlined in the Methods section as it was significantly overexpressed in ASD samples assessed in 8 out of 14 studies. To note, the other six studies have not included RPS4Y1 in the respective lists of assessed signals. Analysis of study heterogeneity showed a significant between-datasets variance of expression levels for this gene $(\mathrm{ISq}=89.46, p \mathrm{Q}>7.74 \mathrm{E}-12$ ), and therefore, a random-effects model was selected. Multiple linear regression analysis indicated that the expression levels of RPS4Y1 were significantly influenced by the sample size and population region (country) ( $p=9.76 \mathrm{E}-3$ and $6.19 \mathrm{E}-6$, respectively), but not by the year when the study was performed $(p=0.94)$. Datasetspecific effect sizes, $95 \%$ confidence intervals, and weights of the gene are shown in Figure 3A.

\section{DISCUSSION}

Previous studies showed that postnatal exposure to PE is a significant risk factor for ASD with mechanistic connections being not yet described $(5,6,13)$. In an attempt to identify novel, not-yet-described molecular pathways that link preeclampsia to subsequently diagnosed ASD phenotypes, we employed natural 

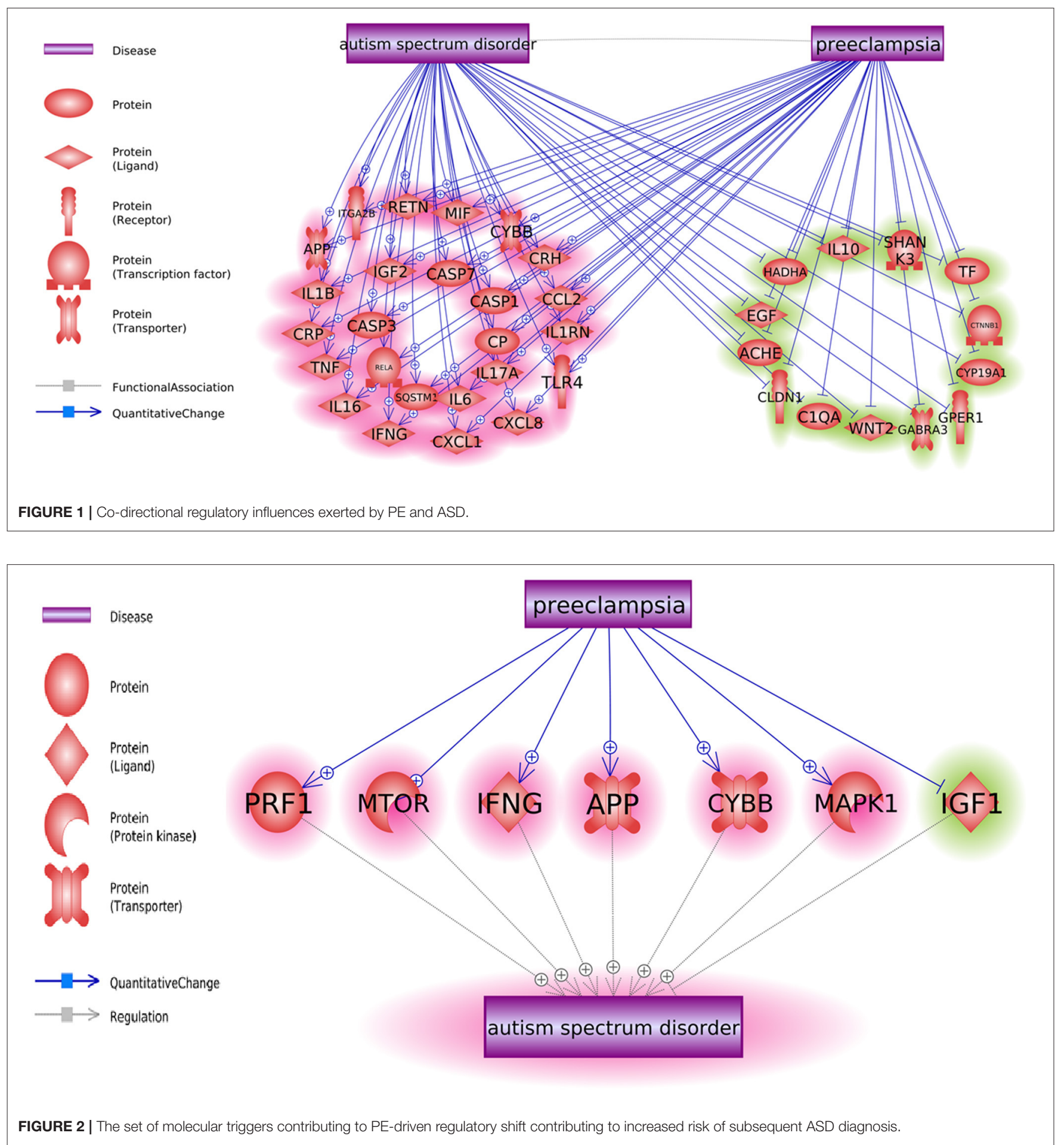

language programming environment Pathway Studio to perform large-scale exploration of previous knowledge concerning both of these conditions.

Notably, sets of genes linked to PE and to ASD demonstrated significant overlaps (see Figure 1, $p=3.14 \mathrm{E}-67$; shared genes: $N=156$ ). This set of shared genes was significantly enriched in the pathways previously implicated with both PE and ASD, such as response to nutrient levels [(14); Sharp et al., 2018], positive regulation of locomotion $(15,16)$, and aging [(17); Ruggieri et al., 2019]. Overall, the results of GSEA suggest that pathophysiological mechanisms of PE and ASD are partially shared. 

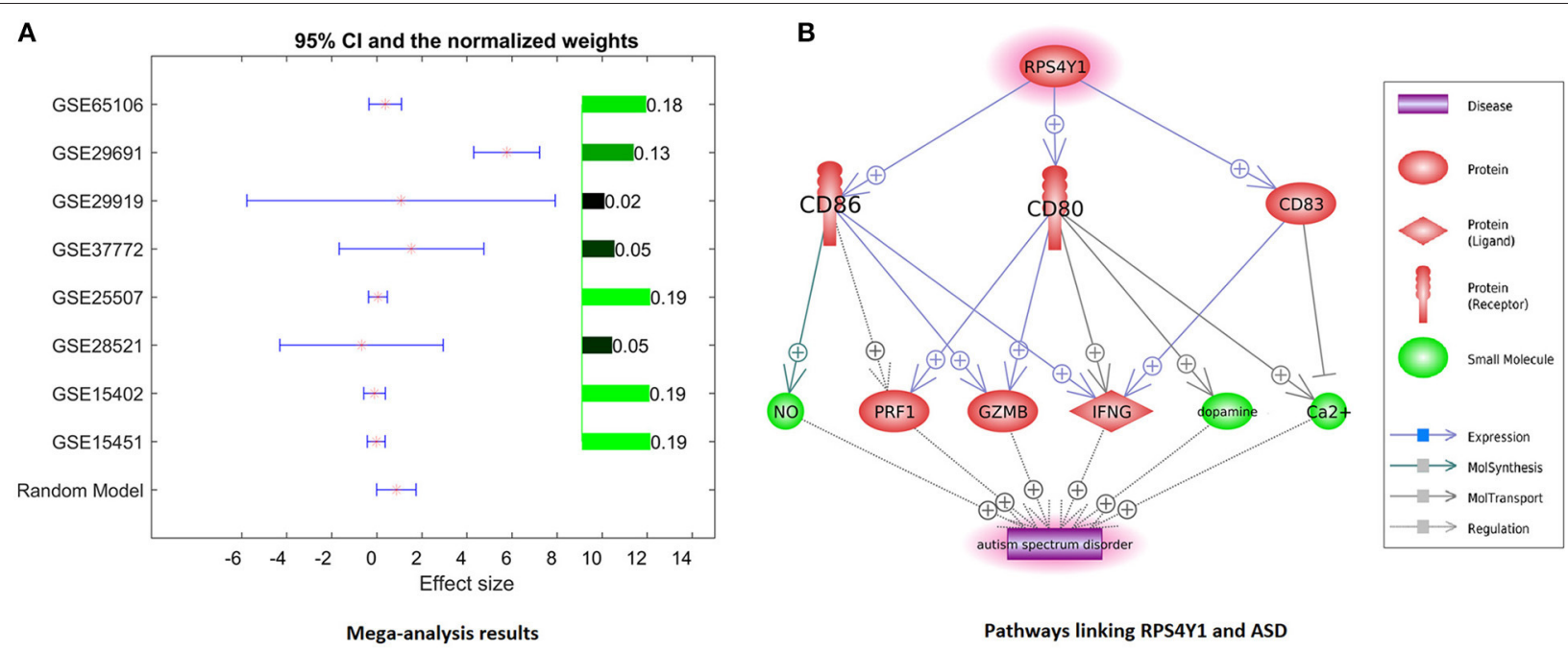

FIGURE 3 | Evidence of preeclampsia-related gene RPS4Y1 involvement in ASD phenotypes. (A) Dataset-specific effect sizes, 95\% confidence intervals, and weights of RPS4Y1. (B) Molecular network connecting RPS4Y1 and ASD.

Some molecules contributing to both PE and ASD were highlighted as molecular triggers set by $\mathrm{PE}$ and possibly contributing to a worsening risk of subsequent ASD (Figure 1). This set includes six ASD-positive regulators that have been stimulated by PE condition and one inhibitor (IGF1) that has been deactivated during PE. Moreover, a bulk of regulatory interactions exerted by PE condition was co-directional with that observed in ASD phenotypes. For example, in PE patients, serum levels of transferrin do decrease (18), a phenomenon suggested as a negative contributor to intrauterine growth (19). At the same time, lower than normal levels of transferrin (Chauhan et al., 2004) or the defects in its saturation (20) are commonly seen in children with ASD diagnosis (21). Similarly, levels of IL6, TNFalpha, and IL17 are repeatedly reported as elevated in both PE and ASD [Kara et al., 2019; (22-27)].

As PE etiologically precedes the diagnosis of ASD, this finding may be interpreted as a PE-driven regulatory shift toward greater vulnerability to the development of ASD, which may occur even by quantifiable at the time of the late pregnancy or delivery (Figures 1, 2).

For further analysis, genes shared between curated PE- and ASD-specific gene sets were removed from consideration in order to ensure that uncovered PE-related contributors to ASD had not been already described as such. A total of 1,032 genes involved in preeclampsia but not in ASD were highlighted by Pathway Studio data mining. In subsequent mega-analysis of expression, each of these genes was investigated for consistent evidence of the changes in their expression in ASD phenotypes across 14 mRNA expression datasets acquired from GEO (Table 1). This analysis highlighted only one novel gene, RPS4Y1, which passed the preselected criterion of the significance of the association. RPS4Y1 is a member of the S4E family of ribosomal proteins, which promotes PE by impairing STAT3 (Signal Transducer and Activator of Transcription 3) signaling and suppressing migration and invasion of trophoblast cells (28). Figure 3B shows that the product of RPS4Y1 may also promote the development of ASD through the activation of multiple membrane proteins, including CD86, CD80, and CD83. In turn, these membrane molecules activate other molecular players previously implicated in ASD, such as ion transport pumps (29), and affect the release of nitric oxide (30). Importantly, all three of these membrane proteins promote the activity of interferon-gamma, which has been suggested to associate with increased oxidative stress in ASD (31), and also being produced at substantially elevated steady-state levels by natural killer (NK) cells of high-functioning adult individuals with ASD (32). Additional information regarding the activation of the IFNG pathway is provided in ASD_PE $\rightarrow$ RPS4Y1_Pathway.

Given the 4:1 male predominance for ASD (33), the involvement of sex-linked genes in these phenotypes is very likely. The RPS4Y gene is located on chromosome $\mathrm{Y}$ and encodes the ubiquitously expressed ribosomal protein gene. Interestingly, the expression of this gene in the process of neural differentiation increases even more (34). XY males express two RPS4-encoding genes (RPS4Y and RPS4X), whereas XX females express only one copy of RPS4X due to X-inactivation; thus, indicating that the levels of RPS4 protein in males vs. females are greater to begin with (35). In XYY individuals, known to score significantly higher on various autism-related scales, RPS4Y is expressed at the levels approximately 2 -fold of that in age-matched, typically developing male controls (36). Exposure to PE may be viewed as yet another way to increase the expression of RPS4Y in affected fetuses systemically. Interestingly, Y-chromosome harbors another autism-related gene, NLGN4Y, which encodes a trans-synaptic cell adhesion molecule that stabilizes excitatory and inhibitory synaptic activity. Gene dosage of NLGN4Y is defined in a manner similar to that of RPS4Y. The involvement of NLGN4Y in autistic phenotypes is widely discussed, as it has been found mutated in some rare family clusters of autism (37). It is likely that the genetic contribution to the predominance of 
autistic males depends on more than one gene, thus justifying the addition of RPS4Y, which is normally expressed at the very high levels throughout human tissues, including the brain, to the list of the candidates in need of further exploration.

There were several limitations of this study that need to be addressed in future work. First, the expression datasets employed in the analysis were lack of age and sex information. The influence of age and sex on the identified ASD potential genes should be explored with relevant data. Second, the pathways connecting RPS4 and ASD were built based on literature data. Experiment data were needed to confirm these relations in the case of ASD (e.g., using co-expression analysis to confirm the gene-gene relations within the pathway).

\section{CONCLUSION}

In conclusion, a large-scale literature mining effort and analysis of expression data allowed us to identify a set of PE-driven molecular triggers, shifting the developing brain toward a risk of ASD. In particular, chromosome Y-encoded gene RPS4Y1, an inhibitor of STAT3 signaling, was highlighted as PE-driven contributor to ASD phenotypes. RPS4Y1 warrants evaluation as a possible contributor to male predominance in ASD.

\section{REFERENCES}

1. Eiland E, Nzerue C, Faulkner M. Preeclampsia 2012. J Pregnan. (2012) 2012:586578. doi: 10.1155/2012/586578

2. Nisar S, Hashem S, Bhat A, Syed N, Yadav S, Azeem M, et al. Association of genes with phenotype in autism spectrum disorder. Aging. (2019) 11:1074270. doi: 10.18632/aging. 102473

3. Rylaarsdam L, Guemez-Gamboa A. Genetic causes and modifiers of autism spectrum disorder. Front Cell Neurosci. (2019) 13:385. doi: 10.3389/fncel.2019.00385

4. Lovato D, Herai R, Pignatari G, Beltrão-Braga P. The relevance of variants with unknown significance for autism spectrum disorder considering the genotype-phenotype interrelationship. Front Psychiatr. (2019) 10:409. doi: $10.3389 /$ fpsyt.2019.00409

5. Dachew B, Mamun A, Maravilla J, Alati R. Pre-eclampsia and the risk of autism-spectrum disorder in offspring: meta-analysis. Br J Psychiatr. (2018) 212:142-7. doi: 10.1192/bjp.2017.27

6. Getahun D, Fassett M, Peltier M, Wing D, Xiang A, Chiu V, et al. Association of perinatal risk factors with autism spectrum disorder. Am J Perinatol. (2017) 34:295-304. doi: 10.1055/s-0036-1597624

7. Driscoll D, Felice V, Kenny L, Boylan G, O'Keeffe G. Mild prenatal hypoxia-ischemia leads to social deficits and central and peripheral inflammation in exposed offspring. Brain Behav Immun. (2018) 69:418-27. doi: 10.1016/j.bbi.2018.01.001

8. Gustafsson H, Sullivan E, Nousen E, Sullivan C, Huang E, Rincon M, et al. Maternal prenatal depression predicts infant negative affect via maternal inflammatory cytokine levels. Brain Behav Immun. (2018) 73:47081. doi: 10.1016/j.bbi.2018.06.011

9. Nikitin A, Egorov S, Daraselia N, Mazo I. Pathway studio-the analysis and navigation of molecular networks. Bioinformatics. (2003) 19:2155-7. doi: 10.1093/bioinformatics/btg290

10. Seifuddin F, Pirooznia M, Judy J, Goes F, Potash J, Zandi P. Systematic review of genome-wide gene expression studies of bipolar disorder. BMC Psychiatr. (2013) 13:213. doi: 10.1186/1471-244X-13-213

11. Borenstein M, Hedges L, Higgins J, Rothstein H. A basic introduction to fixedeffect and random-effects models for meta-analysis. Res Synthes Methods. (2010) 1:97-111. doi: 10.1002/jrsm.12

\section{DATA AVAILABILITY STATEMENT}

Publicly available datasets were analyzed in this study. This data can be found here: gousinfo.com/database/Data_Genetic/ASD_PE.xlsx; www.ncbi. nlm.nih.gov/geo/.

\section{AUTHOR CONTRIBUTIONS}

QX, HC, and FZ designed the study and collected the data. $\mathrm{AB}, \mathrm{SZ}, \mathrm{QX}, \mathrm{ZL}, \mathrm{HC}$, and FZ performed the data analysis and contributed to the writing of the manuscript. AB and SZ provided functional analysis of RPS4Y1 and edited the manuscript into its final shape. QX and FZ contributed to the acquisition of funding that supported this study. All authors read and approved the final manuscript.

\section{FUNDING}

$\mathrm{AB}$ was partially supported by an Egg Nutrition Center (ENCs) grant to study the influence of the choline on ASD-related sensory phenotypes; FZ was partially supported by the National Natural Science Foundation of China (No. 81471364).

12. Reiner A, Yekutieli D, Benjamini Y. Identifying differentially expressed genes using false discovery rate controlling procedures. Bioinformatics. (2003) 19:368-75. doi: 10.1093/bioinformatics/btf877

13. Kim J, Son M, Son C, Radua J, Eisenhut M, Gressier F, et al. Environmental risk factors and biomarkers for autism spectrum disorder: an umbrella review of the evidence. Lancet Psychiatr. (2019) 6:590-600. doi: 10.1016/S2215-0366(19)30181-6

14. Kim J, Kim Y, Lee R, Moon J, Jo I. Serum levels of zinc, calcium, and iron are associated with the risk of preeclampsia in pregnant women. Nutr Res. (2012) 32:764-9. doi: 10.1016/j.nutres.2012.09.007

15. Connolly C, Conger S, Montoye A, Marshall M, Schlaff R, Badon S, et al. Walking for health during pregnancy: a literature review and considerations for future research. J Sport Health Sci. (2019) 8:401-11. doi: 10.1016/j.jshs.2018.11.004

16. Urdaneta $\mathrm{K}$, Castillo $\mathrm{M}$, Montiel N, Semprún-Hernández N, Antonucci N, Siniscalco D. Autism spectrum disorders: potential neuropsychopharmacotherapeutic plant-based drugs. ASSAY Drug Deve Technol. (2018) 16:433-44. doi: 10.1089/adt.2018.848

17. Nakashima A, Tsuda S, Kusabiraki T, Aoki A, Ushijima A, Shima T, et al. Current understanding of autophagy in pregnancy. Int J Mol Sci. (2019) 20:2342. doi: 10.3390/ijms20092342

18. Aksoy H, Taysi S, Altinkaynak K, Bakan E, Bakan N, Kumtepe Y. Antioxidant potential and transferrin, ceruloplasmin, and lipid peroxidation levels in women with preeclampsia. J Investig Med. (2003) 51:284-7. doi: 10.1136/jim-51-05-15

19. Wu Y, Sakamoto H, Kanenishi K, Li J, Khatun R, Hata T. Transferrin microheterogeneity in pregnancies with preeclampsia. Clin Chim Acta. (2003) 332:103-10. doi: 10.1016/s0009-8981(03)00134-7

20. Luck A, Bobst C, Kaltashov I, Mason A. Human serum transferrin: is there a link among autism, high oxalate levels, and iron deficiency anemia? Biochemistry. (2013) 52:8333-41. doi: 10.1021/bi401190m

21. Bjørklund G, Meguid N, El-Ansary A, El-Bana M, Dadar M, Aaseth J, et al. Diagnostic and severity-tracking biomarkers for autism spectrum disorder. $J$ Mol Neurosci. (2018) 66:492-511. doi: 10.1007/s12031-018-1192-1

22. El Shahaway A, Yahia S, Abdelrhman A, Elhady R. Role of maternal serum interleukin 17 in preeclampsia: diagnosis and prognosis. J Inflamm Res. (2019) 12:175-80. doi: 10.2147/JIR.S206800 
23. Daneva AM, Hadži-Lega M, Stefanovic M. Correlation of the system of cytokines in moderate and severe preeclampsia. Clin Exp Obste Gynecol. (2016) 43:220-224. doi: 10.12891/ceog2077.2016

24. Salazar Garcia M, Mobley Y, Henson J, Davies M, Skariah A, Dambaeva $\mathrm{S}$, et al. Early pregnancy immune biomarkers in peripheral blood may predict preeclampsia. J Reprod Immunol. (2018) 125:25-31. doi: 10.1016/j.jri.2017.10.048

25. Black K, Horowitz J. Inflammatory markers and preeclampsia: a systematic review. Nursing Res. (2018) 67:242-51. doi: 10.1097/NNR.00000000000 00285

26. Wei H, Alberts I, Li X. Brain IL-6 and autism. Neuroscience. (2013) 252:320-5. doi: 10.1016/j.neuroscience.2013.08.025

27. Eftekharian M, Ghafouri-Fard S, Noroozi R, Omrani M, Arsang-jang S, Ganji M, et al. Cytokine profile in autistic patients. Cytokine. (2018) 108:120-126. doi: 10.1016/j.cyto.2018.03.034

28. Chen X, Tong C, Li H, Peng W, Li R, Luo X, et al. Dysregulated expression of RPS4Y1 (ribosomal protein S4, Y-linked 1) impairs STAT3 (signal transducer and activator of transcription 3) signaling to suppress trophoblast cell migration and invasion in preeclampsia. Hypertension. (2018) 71:481-90. doi: 10.1161/HYPERTENSIONAHA.117.10250

29. Ji L, Chauhan A, Brown W, Chauhan V. Increased activities of $\mathrm{Na}+/ \mathrm{K}+-$ ATPase and $\mathrm{Ca} 2+/ \mathrm{Mg} 2+-\mathrm{ATPase}$ in the frontal cortex and cerebellum of autistic individuals. Life Sci. (2009) 85:788-93. doi: 10.1016/j.lfs.2009. 10.008

30. Yui K, Kawasaki Y, Yamada H, Ogawa S. Oxidative stress and nitric oxide in autism spectrum disorder and other neuropsychiatric disorders. CNS Neurol Disord Drug Targets. (2016) 15:587-96. doi: 10.2174/1871527315666160413121751

31. Tostes M, Teixeira H, Gattaz W, Brandão M, Raposo N. Altered neurotrophin, neuropeptide, cytokines and nitric oxide levels in autism. Pharmacopsychiatry. (2012) 45:241-3. doi: 10.1055/s-0032-1301914

32. Bennabi M, Tarantino N, Gaman A, Scheid I, Krishnamoorthy R, Debré P, et al. Persistence of dysfunctional natural killer cells in adults with high-functioning autism spectrum disorders: stigma/consequence of unresolved early infectious events? Mol Autism. (2019) 10:22. doi: 10.1186/s13229-019-0269-1

33. Newschaffer C, Croen L, Daniels J, Giarelli E, Grether J, Levy S, et al. The epidemiology of autism spectrum disorders. Ann Rev Pub Health. (2007) 28:235-58. doi: 10.1146/annurev.publhealth.28.021406. 144007

34. Vakilian H, Mirzaei M, Tabar M, Pooyan P, Rezaee L, Parker L, et al. DDX3Y, a male-specific region of $\mathrm{Y}$ chromosome gene, may modulate neuronal differentiation. J Proteome Res. (2015) 14:3474-83. doi: 10.1021/acs.jproteome.5b00512

35. Staedtler F, Hartmann N, Letzkus M, Bongiovanni S, Scherer A, Marc P, et al. Robust and tissue-independent gender-specific transcript biomarkers. Biomarkers. (2013) 18:436-45. doi: 10.3109/1354750X.2013.811538

36. Ross J, Tartaglia N, Merry D, Dalva M, Zinn A. Behavioral phenotypes in males with XYY and possible role of increased NLGN4Y expression in autism features: NLGN4Y overexpression in XYY. Genes Brain Behav. (2015) 14:137-44. doi: 10.1111/gbb.12200

37. Yan J, Noltner K, Feng J, Li W, Schroer R, Skinner C, et al. Neurexin $1 \alpha$ structural variants associated with autism. Neurosci. Lett. (2008) 438:368-70. doi: 10.1016/j.neulet.2008.04.074

Conflict of Interest: The authors declare that the research was conducted in the absence of any commercial or financial relationships that could be construed as a potential conflict of interest.

Copyright (c) 2020 Xie, Li, Wang, Zaidi, Baranova, Zhang and Cao. This is an open-access article distributed under the terms of the Creative Commons Attribution License (CC BY). The use, distribution or reproduction in other forums is permitted, provided the original author(s) and the copyright owner(s) are credited and that the original publication in this journal is cited, in accordance with accepted academic practice. No use, distribution or reproduction is permitted which does not comply with these terms. 